\title{
THE HOMOTOPY CONTINUATION METHOD: NUMERICALLY IMPLEMENTABLE TOPOLOGICAL PROCEDURES
}

\author{
BY \\ J. C. ALEXANDER ${ }^{1}$ AND JAMES A. YORKE ${ }^{1}$
}

\begin{abstract}
The homotopy continuation method involves numerically finding the solution of a problem by starting from the solution of a known problem and continuing the solution as the known problem is homotoped to the given problem. The process is axiomatized and an algebraic topological condition is given that guarantees the method will work. A number of examples are presented that involve fixed points, zeroes of maps, singularities of vector fields, and bifurcation. As an adjunct, proofs using differential rather than algebraic techniques are given for the Borsuk-Ulam Theorem and the Rabinowitz Bifurcation Theorem.
\end{abstract}

In 1963, Hirsch [13] gave a short-and by now classic-proof of the Brouwer Fixed Point Theorem by a generic argument. This theorem is usually proved by some kind of degree argument and degree generically counts inverse images of points. Hirsch directly looked at the inverse image of a generic point. The idea that one could replace degree arguments by looking at the inverse images of points of maps was made the theme of a book by J. Milnor [24] and later by V. Guillemin and A. Pollack [12, especially Chapters 2, 3] and Hirsch [14, especially Chapter 5]. We offer these books as general references for transversality and Sard's theorem.

Without knowledge of Hirsch's paper, H. Scarf [31] in 1967 used much the same ideas to numerically approximate a Brouwer fixed point. One "follows" a "path" which leads from the boundary to some one or more of the fixed points. For some contemporary papers using similar methods, see [19], [23]. This has been called the Newton method. B. C. Eaves [8] in 1972 developed a slightly different approach to the same end. His idea was to homotope one of a set of standard maps to the map in question; by following the fixed points of the changing maps, the fixed-point-set of the map in question could be located. It is this version we call the homotopy continuation method and

Received by the editors April 5, 1977.

AMS (MOS) subject classifications (1970). Primary 68A10; Secondary 55B20, 54H25, 55C20, 55C25, 47M10, 57D35, 34A15.

Key words and phrases. Homotopy continuation method, Newton method, generic approximation, generic proofs, fixed points, bifurcation, vector fields, Borsuk-Ulam Theorem.

${ }^{1}$ Partially supported by NSF Grants. 
develop rigorously. Eaves and Scarf jointly refined their methods in [10].

Influenced by Milnor's book, and independently of Scarf and Eaves, R. B. Kellogg, T. Y. Li, and J. A. Yorke [16] reported in 1974 on a numerical method similar to that of Scarf. They implemented the process on a UNIVAC 1108; for a 20-dimensional problem, the machine required slightly over three seconds to locate a fixed point [17]. Meanwhile, S. Smale used some of Scarf's ideas to find equilibrium points of economic systems [32], and he and Hirsch generalized the method to find zeroes of maps (unpublished). Yorke learned of Eaves' homotopy approach and realized it could be made rigorous and applied to a variety of problems. This was explicated by S. N. Chow, J. Mallet-Paret and Yorke in [7].

For expositions of some of these methods, see [9] and [29], and for discussions of related technical questions, see [20], [21], [28] and [30].

The underlying idea of both the Newton and homotopy methods is that the inverse image of a generic point is a path that can be considered, say, the solution of a differential equation. It can then be followed by any of a number of numerical techniques and the fixed point set can be numerically tracked down. The purpose of this paper is to generalize (rigorously) the method so it works for other problems. The homotopy method is known to work for problems which are known to have a solution by a degree argument. To a topologist, degree is an obstruction lying in some homology group. Here we free the method of its dependence on degree; if a solution of the problem is guaranteed by any obstruction lying in some generalized homology or cohomology, the homotopy method will work. However, it may nơt be that the solution set is generically zero dimensional. Thus the set we want to follow through the homotopy may not be a one-dimensional curve; it may be a higher-dimensional manifold or stratified set. Both of these generalizations were forced on us by the vector-field problem on spheres. In turn, the vector-field problem is interesting because it offers an approach to locating bifurcating sets of a nonlinear equation. We consider this problem in this paper. The number of vector fields to be considered is the dimension of the parameter space in the bifurcation problem; thus the classical case of a one-dimensional parameter is connected to the problem of one vector field on an even-dimensional sphere. This was recognized in [7]. With two vector fields, one can tackle Hopf bifurcation. See [4]. For a one-dimensional parameter, there are a number of other approaches that have been developed for this important problem. We refer the reader to [18] and its bibliography.

In our first section, we develop the connection between algebraic topology and the continuation method. We then lay out the general format of the method. In the early papers, a simplicial or piecewise-linear method was used. In the later papers of Smale, Kellogg, $\mathrm{Li}$ and Yorke, etc., differential methods 
were used. In our format, it matters not. One can work in either category although, for definitiveness, we consider the differentiable case. We also show how the method is applicable in a number of concrete examples.

In some cases, particularly for problems that can be solved by classical degree theory, one is able to extract a proof of the existence of a solution to the problem from a continuation argument. For example, this is usually the case for fixed-point problems. In the present paper, we give a very short "generic" proof of a form of the Borsuk-Ulam Theorem. Carrying this idea further, we swing full circle to Hirsch's original concept, and, as an adjoint, present nonalgebraic proofs of topological theorems. We give differential topology proofs of P. Rabinowitz' bifurcation theorem and complete $\mathrm{L}$. Nirenberg's approach to the Borsuk-Ulam Theorem.

An algebraic condition. We will consider maps from a manifold $M$ with boundary $\partial M$ to another manifold $N$ containing some closed subset $P$. Suppose $f:(M, \partial M) \rightarrow(N, N-P)$ is some map which cannot be deformed off $P$. (I.e. if $f^{\prime}:(M, \partial M) \rightarrow(N, N-P)$ is any map which is homotopic to $f$ via a homotopy that keeps $\partial M$ in $N-P$, then $f^{\prime}(M) \cap P \neq \varnothing$.) Let $F$ : $(M \times I, \partial M \times I) \rightarrow(N, N-P)$ be some deformation of $f$, where $I$ is the unit interval. Let $M_{i}=M \times\{i\} \subset M \times I$. The question we ask is: Does $F^{-1}(P)$ necessarily connect $M_{0}$ and $M_{1}$ ? We are indebted to John Guckenheimer for the following example where, in fact, $F^{-1}(P)$ cannot connect $M_{0}$ and $M_{1}$. The Borromean rings are a well-known configuration of three circles in $R^{3}$ which are linked but such that any two can be separated. Let $M$ be a disk with one of the rings as boundary. Let $N=R^{3}$ and $P$ be the other two rings. The disk can be situated so as to intersect precisely one of the other two rings (by bulging in one direction or the other). Let $f$ and $f^{\prime}$ realize these two cases. Then $f$ can easily be deformed to $f^{\prime}$ and $F^{-1}(P)$ cannot connect $M_{0}$ and $M_{1}$ since the two circles making up $P$ are not connected. A similar example where $F^{-1}(P)$ need not connect $M_{0}$ and $M_{1}$ with $P$ connected is given in the figure. Here $N=R^{3}, P$ is a circle as indicated, and $M$ is a disk with the other circle as boundary. The maps $f$ and $f^{\prime}$ realize the disk with a bulge to the left and right and $F$ deforms $f$ to $f^{\prime}$ linearly.
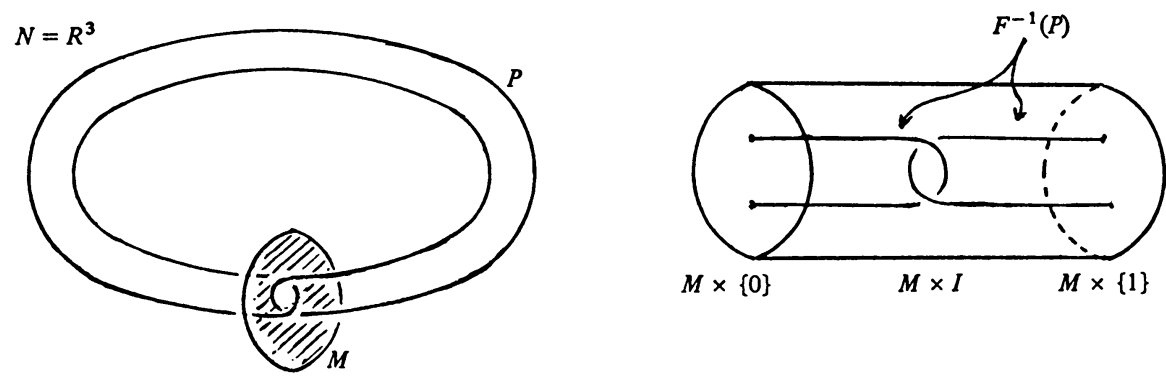
The continuation method works when $F^{-1}(P)$ does connect $M_{0}$ and $M_{1}$. We offer the following useful pair of sufficient conditions, which subsume all previous examples. Consider the two dual conditions:

(A) $f_{*}: h_{*}(M, \partial M) \rightarrow h_{*}(N, N-P)$, or

$\left(\mathrm{A}^{\prime}\right) f^{*}: h^{*}(N, N-P) \rightarrow h^{*}(M, \partial M)$ is not the zero map for some (generalized) homology or cohomology $h$.

We claim that if $(\mathrm{A})$ or $\left(\mathrm{A}^{\prime}\right)$ obtains, then $F^{-1}(P)$ connects $M_{0}$ and $M_{1}$ for any deformation $F$. Consider $\left(\mathrm{A}^{\prime}\right)$. Suppose that $F^{-1}(P)$ is the disjoint union of two closed subsets $Q_{0}$ and $Q_{1}$ in $M \times I$ where $Q_{0} \cap M_{0}=\varnothing$ and $Q_{1} \cap M_{1}=\varnothing$. Let $j_{i}: M \rightarrow M \times I$ be the inclusion of $M$ as $M_{i}$ for $i=0,1$. Consider

$$
F:\left(M \times I, M \times I-F^{-1}(P)\right) \rightarrow(N, N-P) ;
$$

clearly $F j_{0}=f$. Now

$$
j_{i}^{*}: h^{*}\left(M \times I, M \times I-Q_{i}\right) \rightarrow h^{*}(M, \partial M)
$$

is the zero map since $j_{i}(M) \subset M \times I-Q_{i}$. Moreover,

$$
j_{0}^{*}: h^{*}\left(M \times I, M \times I-Q_{1}\right) \rightarrow h^{*}(M, \partial M)
$$

is also the zero map, since $j_{0}, j_{1}:(M, \partial M) \rightarrow\left(M \times I, M \times I-Q_{1}\right)$ are homotopic. On the other hand, the map

$$
\begin{aligned}
& h^{*}(M \times I,\left.M \times I-Q_{0}\right) \oplus h^{*}\left(M \times I, M \times I-Q_{1}\right) \\
& \rightarrow h^{*}\left(M \times I, M \times I-F^{-1}(P)\right)
\end{aligned}
$$

induced by the inclusions is an isomorphism by the relative Mayer-Vietoris sequence and, therefore,

$$
j_{0}^{*}: h^{*}\left(M \times I, M \times I-F^{-1}(P)\right) \rightarrow h^{*}(M, \partial M)
$$

is zero. Hence $f^{*}=j_{0}^{*} F^{*}$ is zero and the claim is proved.

The general setup. As above, $M$ is a manifold with boundary (possibly empty) and $N$ is another manifold, assumed without boundary. We assume $P$ is a stratified set in $N$. A stratified set is one which is the disjoint union of submanifolds which fit together nicely. See [22, Chapter 5] for a precise definition and discussion of the concept. In particular, it has a nice neighborhood in $N$ we can call a tubular neighborhood. In many cases, $P$ is a submanifold. We work in a category of manifolds and maps where there is a good concept of transversality and where transversality theorems hold. We have in mind either the smooth or piecewise linear category. A map $f$ : $(M, \partial M) \rightarrow(N, N-P)$ is called proper to $P$ if for some tubular neighborhood $W$ of $P$, the inverse image $f^{-1}(\bar{W})$ is compact and disjoint from $\partial M$. A homotopy $F:(M \times I, \partial M \times I) \rightarrow(N, N-P)$ is proper to $P$ if $F^{-1}(\bar{W})$ is compact and disjoint from $\partial M \times I$ for some tubular neighborhood $W$ of $P$. 
We suppose we are given an $f:(M, \partial M) \rightarrow(N, N-P)$ proper to $P$. We also need a parameter manifold $A$ without boundary. Suppose we have a map $\Phi$ : $(A \times M \times I, A \times \partial M \times I) \rightarrow(N, N-P)$ satisfying the following conditions:

$\Phi 1 . \Phi$ is transverse to $P$, except possibly for $t=1$.

$\Phi 2 . \Phi(\alpha, \cdot, 1):(M, \partial M) \rightarrow(N, N-P)$ is $f:(M, \partial M) \rightarrow(N, N-P)$ for all $\alpha \in A$.

$\Phi 3 . \Phi(\alpha, \cdot, \cdot):(M \times I, \partial M \times I) \rightarrow(N, N-P)$ is proper to $P$ as a homotopy for all $\alpha \in A$.

For $\alpha \in A$, we denote $\Phi(\alpha, \cdot, 0):(M, \partial M) \rightarrow(N, N-P)$ by $f_{\alpha}$ and $\Phi(\alpha, \cdot, \cdot):(M \times I, \partial M \times I) \rightarrow(N, N-P)$ by $\Phi_{\alpha}$.

For $\varepsilon \geqslant 0$, let

$$
R_{\varepsilon}=\left\{\alpha \in A\left|\Phi_{\alpha}\right| M \times[0,1-\varepsilon] \text { and } f_{\alpha} \text { are transverse to } P\right\} .
$$

Let

$$
R^{0}=\bigcap_{\varepsilon>0} R_{\varepsilon}=\left\{\alpha \in A\left|\Phi_{\alpha}\right| M \times[0,1) \text { and } f_{\alpha} \text { are transverse to } R\right\} .
$$

Let $Q_{\alpha}=\Phi_{\alpha}^{-1}(P)$. For $\alpha \in R^{0}$, the set $Q_{\alpha}$ is a stratified set in $M \times I$ intersecting $M_{0}$ transversally. If $P$ is a manifold, so is $Q_{\alpha}$. The dimension of $Q_{\alpha}$ equals

$$
\text { dimension } M+\text { dimension } P-\text { dimension } N+1 \text {. }
$$

That is, the codimension of $Q_{\alpha}$ in $M \times I$ equals the codimension of $P$ in $N$. In fact the inverse image of some tubular neighborhood $W$ of $P$ in $N$ is a tubular neighborhood of $Q_{\alpha}$ in $M \times I$. This tubular neighborhood is a key ingredient for the continuation method.

Recall that a generic subset of a manifold is one containing an open dense subset. A residual subset is the countable intersection of generic subsets. By the Baire Category Theorem, a residual subset is dense. Since for the continuation method one chooses random points of $A$, the probability aspects of a set may be more important. We define a subset to be full if its complement has measure zero. The fundamental lemma about transversality is the following: suppose a map from the product of two manifolds is transverse to some submanifold (or stratified set) $P$ of the range; then for a full residual subset of points $\alpha$ of the first manifold of the product, the map restricted to the $\alpha$-slice is transverse to $P$. The next several results are straightforward applications of the fundamental lemma, and we leave the proofs to the reader. For $\alpha \in A$, let $Q=Q_{\alpha}=\Phi_{\alpha}^{-1}(P)$.

1. The set $R^{0}$ is residual and full in $A$. Thus for $\alpha \in R^{0}$ and $\varepsilon>0$, the set $Q_{\alpha} \cap(M \times[0,1-\varepsilon])$ is a compact stratified set (with pull-back tubular neighborhood) intersecting $M_{0}$ transversally. 
In particular applications, more information may be available. We state two common conditions and their consequences.

(T) The given map $f:(M, \partial M) \rightarrow(N, N-P)$ is transverse to $P$.

(C) The manifold $M$ is compact.

As to the consequences:

2. If (T) holds, the set $R_{0}$ is residual and full. For $\alpha \in R_{0}$, the set $Q_{\alpha}$ is a compact stratified set intersecting $M_{0}$ and $M_{1}$ transversally.

3. If (C) holds, for each $\varepsilon>0$, the set $R_{\varepsilon}$ is generic and full.

4. If (T) and (C) hold, the set $R_{0}$ is generic and full.

The reader is invited to show that if $P$ is a manifold, for a residual set of $\alpha \in A$, the projection $Q_{\alpha} \subset M \times I \rightarrow I$ is a Morse function on $Q_{\alpha}$ (at least if (T) holds). See [25], particularly $\$ 6$. The reader is also invited to generalize this if $P$ is a stratified set.

We now consider a common method of constructing $\Phi$. Often, the manifold $N$ has some kind of linear structure and maps can be added pointwise. For example, $N$ is Euclidean space or a vector bundle over $M$ (in which case the category is of cross-sections). We start with some collection of $f_{\alpha}$ : $(M, \partial M) \rightarrow(N, N-P)$ (continuous on $A \times M)$ and let

$$
\Phi(\alpha, m, t)=t f(m)+(1-t) f_{\alpha}(m)
$$

Let $F: A \times M \rightarrow N$ be the union of the $f_{\alpha}$. Consider the condition:

(S) For each $m$ in $M-\partial M$, the map $A \rightarrow N$ defined by $\alpha \rightarrow F(\alpha, m)$ is a submersion (i.e. the differential is onto).

5. If (S) holds and each $\Phi_{\alpha}$ (as well as $f$ ) is proper to $P$, then $\Phi$ satisfies $\Phi 1-\Phi 3$.

The proof is trivial. One can obviously weaken (S) and still get a result like (5), but we have stated (S) since it is a common and easily verifiable condition.

The continuation method. We will not develop an algorithm based on the previous setup, but we would like to explain the philosophy behind any algorithm. Suppose we are given a map $f:(M, \partial M) \rightarrow(N, N-P)$ satisfying (A) or (A') and a map $\Phi: A \times M \times I \rightarrow N$ satisfying $\Phi 1-\Phi 3$. The maps $f_{\alpha}$ : $(M, \partial M) \rightarrow(N, N-P)$ are supposed to be "standard" in that each $f_{\alpha}^{-1}(P)$ is supposed to be completely known. With probability one, a choice of $\alpha \in A$ will yield a stratified set (or manifold) $Q_{\alpha}$ in $M \times I$ which connects $M_{0}$ and $M_{1}$ (because of $(\mathrm{A})$ or $\left(\mathrm{A}^{\prime}\right)$ ). The idea is to trace out $Q_{\alpha}$ in $M \times I$ starting from the known $Q_{\alpha} \cap M_{0}$ and ending at the desired $Q_{\alpha} \cap M_{1}$. Moreover, since $Q_{\alpha} \cap[0,1-\varepsilon]$ is compact for each $\varepsilon>0$, in a finite time one can get within $\varepsilon$ of $M_{1}$. If (T) holds, one can get all the way to $M_{1}$ in a finite time. The 
best way to trace out $Q_{\alpha}$ depends on the particular problem, but the following information is available. At each point of $Q_{\alpha}$, its tangent space is known. Thus $Q_{\alpha}$ is known nearby to first order. A step is taken in the tangent space. Although one does not expect to hit $Q_{\alpha}$ with this step, at least one can land within the tubular neighborhood with which $Q_{\alpha}$ comes. This allows a correction to be made; i.e. the fiber can be followed back to the zero section $Q_{\alpha}$. Moreover, one often has information about the curvature of $Q_{\alpha}$ from the second derivative of $\Phi_{\alpha}$, and the optimum size of the step can be estimated. See [20], [21].

In problems detected by degree, $Q_{\alpha}$ is one dimensional, i.e. a curve. If everything is oriented, as is usually the case, the tubular neighborhood is a trivial bundle and there is a differential equation defined on it for which $Q_{\alpha}$ is a solution. Thus the numerical techniques for solving differential equations can be brought to bear. This was done in [17].

Applications. We present a number of examples in this section which can be handled by the previous methods. The reader is referred to [7] for some others. We hope the reader will be convinced the generality we have developed in the previous sections is not unnecessarily abstract.

1. Brouwer fixed point. Let $M=\mathrm{a}$ closed disk $D^{n}$ in $R^{n}, N=R^{n}$ and $A=$ interior of $D^{n}$, and $P=\{0\}$. Suppose $g: D^{n} \rightarrow D^{n}$ is some continuous map. Let $f: D^{n} \rightarrow R^{n}$ be $f(m)=m-g(m)$. For each $\alpha \in A$, let $f_{\alpha}(m)=\alpha$ - $m$ and define $\Phi$ using (H). It is well known that $f^{*}: H^{n}\left(R^{n}, R^{n}-\{0\}\right) \rightarrow$ $H^{n}\left(D^{n}, \partial D^{n}\right)$ is an isomorphism and thus $\left(\mathrm{A}^{\prime}\right)$ holds. Alternatively one can bypass $\left(\mathrm{A}^{\prime}\right)$ and extract a proof of the fixed-point theorem from the setup, as in [7]. Note that the dimension of $Q_{\alpha}$ is one for $\alpha \in R^{0}$.

2. Fixed points of self maps. Let $M$ be any closed connected compact manifold. Let $N=M \times M$ and $P$ be the diagonal $\Delta \subset M \times M$. For any map $g: M \rightarrow M$ with nonzero Lefschetz number $\Lambda$, define $f: M \rightarrow N=M \times$ $M$ by $f(m)=(m, g(m))$. Thus $f^{-1}(\Delta)$ is the set of fixed points of $g$. Moreover, $H^{n}(M \times M, M \times M-\Delta)$ has a canonical generator $\delta$ (called the diagonal class) and $f^{*}(\delta)=\Lambda \cdot$ generator of $H^{n}(M)$. Hence $\left(\mathrm{A}^{\prime}\right)$ holds. Here, of course, the choice of $A$ and $\Phi$ depends on $M$. Again $\operatorname{dim} Q_{\alpha}=1$, for $\alpha \in R^{0}$.

3. Zeroes of monotone functions (Hirsch-Smale). Let $f: R^{n} \rightarrow R^{n}$ be any proper map such that $f(x) \cdot x>0$ outside some disk $D^{n}$. Let $M=D^{n}$, $N=R^{n}$, and $P=\{0\}$. On the boundary of $D^{n}$, the map $f$ is homotopic to the identity map; hence $f^{*}: H^{n}\left(R^{n}, R^{n}-\{0\}\right) \rightarrow H^{n}\left(D^{n}, \partial D^{n}\right)$ is an isomorphism (so ( $\left(\mathrm{A}^{\prime}\right)$ holds). Let $A$ be some disk centered at the origin in $R^{n}$, let $f_{\alpha}(m)=m-\alpha$ and define $\Phi$ by $(\mathrm{H})$. Again $Q_{\alpha}$ has dimension one for $\alpha \in R^{0}$.

4. Roots of complex polynomials. Let $f$ be some complex polynomial of degree $n$. Let $M$ be some disk in the complex numbers $C$ which by a priori 
estimates is known to contain all the roots of $f$. Let $N=C$ (the range of $f$ ) and $P=\{0\}$. Then $f^{*}: H^{2}(C, C-\{0\}) \rightarrow H^{2}(M, \partial M)$ maps the generator to $n$ times the generator. Let $A=C^{n}$ and define $f(z)=\left(z-z_{1}\right) \cdots\left(z-z_{n}\right)$ if $\alpha=\left(z_{1}, \ldots, z_{n}\right) \in C^{n}$. Define $\Phi$ using $(\mathrm{H})$. As above, the dimension of $Q_{\alpha}$ is one for $\alpha \in R^{0}$.

5. Inverse eigenvalue problem. Let $m$ be some $n$-by- $n$ complex matrix. The problem is to find a diagonal matrix $\Delta$ (with entries $d_{1}, \ldots, d_{n}$ ) such that $m+\Delta$ has prescribed eigenvalues-or what is the same, a prescribed characteristic polynomial $t^{n}-\bar{a}_{1} t^{n-1}+\cdots+(-1)^{n} \bar{a}_{n}$. Write $\bar{a}=\left(\bar{a}_{1}, \ldots, \bar{a}_{n}\right)$ $\in C^{n}$. A priori, one can find a disk $D^{2 n} \subset C^{n}$ so that $\left(d_{1}, \ldots, d_{n}\right)$ must lie in $D^{2 n}$. Let $M$ be this disk $D^{2 n}$ of diagonal matrices $\Delta$. Let $N=C^{n}$ and define $f(\Delta): M \rightarrow N$ by $f(\Delta)=\left(a_{1}, \ldots, a_{n}\right)$ if the characteristic polynomial of $m+\Delta$ is $t^{n}-a_{1} t^{n-1}+\cdots+(-1)^{n} a_{n}$. We want to solve $f(\Delta)=\bar{a}$ for $\Delta$. Let $P=\{\bar{a}\} \subset C^{n}$. Then $f^{*}: H^{n}\left(C^{n}, C^{n}-P\right) \rightarrow H^{n}(M, \partial M)$ maps the generator to $n$ ! times a generator [3]. Let $A \subset C^{n^{2}}$ be a disk of $n$-by- $n$ matrices containing $m$ and the zero matrix. Using $(\mathrm{H})$ to define $\Phi$, we let $f_{\alpha}(\Delta)$ equal the coefficients of the characteristic polynomial of $m-\alpha+\Delta$ (work near $\alpha=m$ ). Again $Q_{\alpha}$ is one dimensional for $\alpha \in R^{0}$.

This problem is more precisely called the additive inverse eigenvalue problem. S. Friedland has also studied the related multiplicative inverse eigenvalue problem (where one tries to specify the eigenvalues of $m \Delta$ ) by degree methods [11]. Thus the multiplicative problem should also be amenable to the continuation method.

6. Zeroes of odd maps. The Borsuk-Ulam Theorem has several manifestations, one of which states that any odd map of an $n$-sphere $S^{n}$ to $R^{n}$ (i.e. $f(-x)=-f(x))$ has a zero. Let $M=S^{n}, N=R^{n}$, and $P=\{0\}$. Let $A$ be the special orthogonal group $\mathrm{SO}(n+1)$, and for $\alpha \in A$, let $f_{\alpha}: S^{n} \rightarrow R^{n}$ be the rotation of the sphere by $\alpha$ followed by projection down to $R^{n}$, and define $\Phi$ by $(\mathrm{H})$. In this case, it turns out to be easier to bypass (A) and (A'). For $\alpha \in R^{0}$, the set $Q_{\alpha}$ is a smooth one-dimensional curve in $S^{n} \times I$ intersecting $M_{0}=S^{n}$ at two points $p$ and $-p$, and such that if $(x, t) \in S^{n} \times I$ is in $Q_{\alpha}$ so is $(-x, t)$. We shall show that $Q_{\alpha} \cap\left(S^{n} \times I\right)$ is not empty, which will prove the result. If $Q_{\alpha}$ does not connect $M_{0}$ and $M_{1}$, then some component of $Q_{\alpha}$ is a compact smooth curve $C_{\alpha}$ which must connect $p$ and $-p$. Since $C_{\alpha}$ is a smooth or piecewise-linear curve, it can be parameterized by arc length $s$. Denote the points of $C_{\alpha}$ by $(p(s), t(s))$ where $p(s) \in S^{n}$ and $t(s) \in I$. Thus $p(0)=p, p(\bar{s})=-p$, where $\bar{s}$ is the length of $C_{\alpha}$. Moreover $p(s)=-p(\bar{s}-$ $s)$, since $\Phi_{\alpha}$ is odd. Thus $p\left(\frac{1}{2} s\right)=-p\left(\frac{1}{2} s\right)$, which cannot be. Thus $Q_{\alpha}$ connects $M_{0}$ and $M_{1}$. Note that this in fact constitutes a proof of the Borsuk-Ulam Theorem as we have stated it. This proof generalizes immediately to manifolds more general than $S^{n}$ and group actions more general 
than the $Z / 2 Z$ antipodal action. We will consider another generic proof of the Borsuk-Ulam Theorem in the next section.

7. Dependencies of vector fields on spheres. All the previous examples have been detected by degree in ordinary homology and the manifolds $Q_{\alpha}$ have been one dimensional. This is an example with neither of these attributes. Let $S^{n-1}$ be the unit sphere in $R^{n}$. There is a limit to the number of linearly independent vector fields $S^{n-1}$ can support. For example, an even-dimensional sphere cannot support any. Write $n=$ odd $\times 2^{c} 16^{d}$ where $c=0,1,2$, 3. Let $\rho=\rho_{n}$ (the Hurewitz-Radon number) be $2^{c}+8 d$. Then $S^{n-1}$ admits $\rho-1$ linearly independent vector fields, but not $\rho$ [1]. Suppose there are $\rho$ vector fields on $S^{n-1}$; we want to locate the points on $S^{n-1}$ where they are dependent.

Let $M=S^{n-1}$ and let $N$ be the $\rho$-fold Whitney sum of the tangent bundle of $M$. A cross-section $s$ of $N$ is an ordered set $\left(s_{1}, \ldots, s_{\rho}\right)$ of vector fields. Let $P$ be the subbundle of $N$ with each fiber the $\rho$-fold tangent vectors which are not linearly independent. Thus we are interested in the set of $x \in S^{n-1}$ with $s(x) \in P$. If $n-1$ is odd, $P$ is not a manifold, but it is a stratified set. See [7] for the case of $n-1$ even. The largest stratum of $P$ is the set of $\rho$-tuples of vectors which span a $(\rho-1)$-dimensional space and the lower strata consist of $\rho$-tuples which span spaces of lesser dimensions. The codimension of $P$ in $N$ is $n-\rho$, so the dimension of any $Q_{\alpha}$ is $\rho$. Note that the second stratum of $P$ has codimension larger than $n-1$, so for $\alpha \in R^{0}$, the image of $\Phi_{\alpha}$ on $M \times[0,1)$ intersects only the top stratum of $P$. Thus, although $P$ is a stratified set, $Q_{\alpha} \cap(M \times[0,1))$ is a manifold. Put another way, over $Q_{\alpha} \cap$ $(M \times[0,1))$, there is exactly one dependency. There are "standard" $(\rho-1)$ fold fields on $S^{n-1}$ (see e.g. [33]); presumably one more can be found which has a nice set of dependencies with the others (a sub $S^{\rho-1}$ ?). Let $A$ be a neighborhood of the identity in the $\rho$-fold product $\mathrm{SO}(n) \times \cdots \times \mathrm{SO}(n)$, and let $S_{\alpha}$ be the $\rho$-field with the $i$ th component rotated by the $i$ th component of $\alpha$. Define $\Phi$ using $(\mathrm{H})$.

It remains to verify (A). Let $\pi: N \rightarrow S^{n-1}$ be the projection. Since $N$ is a vector bundle, $\pi$ is a homotopy equivalence. Let $V=V_{n, \rho+1}$ be the Stiefel manifold of orthonormal $(\rho+1)$-frames in $n$ space. That is,

$$
V=\left\{\left(v_{0}, \ldots, v_{\rho}\right) \mid v_{i} \text { is unit vector in } R^{n} \text { and } v_{i} \perp v_{j} \text { if } i \neq j\right\} .
$$

Let $\pi^{\prime}: V \rightarrow S^{n-1}$ be $\pi^{\prime}:\left(v_{0}, \ldots, v_{\rho}\right) \mapsto v_{0}$. The Gram-Schmidt orthonormalization process shows that $V$ is a deformation retract of $N-P$. Let $s$ : $S^{n-1} \rightarrow N$ be any cross-section. We have the following commutative diagram with the horizontal row exact. 


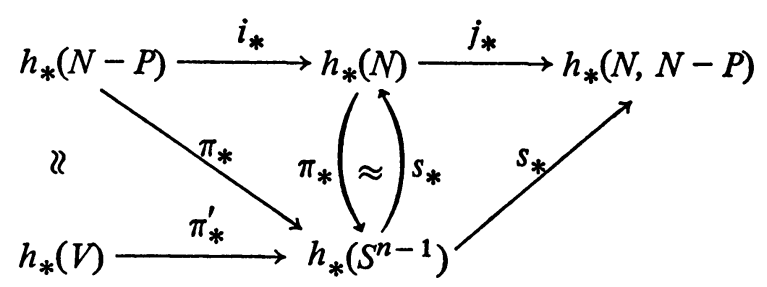

If $s_{*}: h_{*}\left(S^{n-1}\right) \rightarrow h_{*}(N, N-P)$ is the zero map, then $i_{*}: h_{*}(N-P) \rightarrow$ $h_{*}(N)$ is surjective; hence so is $\pi_{*}^{\prime}: h_{*}(N-P) \rightarrow h_{*}\left(S^{n-1}\right)$. Let $h_{*}$ be stable homotopy. The top dimensional cell of $V$ is $(n-1)$-dimensional and if $\pi_{*}^{\prime}$ : $h_{*}(N-P) \rightarrow h_{*}\left(S^{n-1}\right)$ is surjective, there exists a stable map $\sigma: S^{n-1} \rightarrow V$ such that $\pi_{*}^{\prime} \sigma_{*}: h_{*}\left(S^{n-1}\right) \rightarrow h_{*}\left(S^{n-1}\right)$ is an isomorphism. In topological jargon, $V$ is $S$-reducible. However the whole thrust of the solution of the vector field problem is showing that $V$ is not $S$-reducible (see [15, Chapter $15])$. Thus we have a contradiction and (A) is valid.

The fact that dependencies of vector fields are amenable to the methods of this paper is applicable to certain bifurcation problems. Let $B$ be a Banach space and let $F: R^{n} \times B \rightarrow B$ be a continuous map. Denote the points of $R^{n}$ and $B$ by $\lambda, x$, respectively. If $B$ is infinite dimensional, we require that $F$ is of the form (identity + compact). We hypothesize that $F(\lambda, 0)=0$ and denote the derivative $D_{x} F(\lambda, 0)=L(\lambda)$, which we can expand in a first-order Taylor series around $\lambda=0$ :

$$
L(\lambda)=l_{0}+\lambda \cdot l_{1}+O\left(|\lambda|^{2}\right) .
$$

We suppose that $L(\lambda)$ is invertible for $\lambda \neq 0$ at least in a neighborhood of 0 , and that there exists $\varepsilon>0$ such that

$$
\left|\left(l_{0}+\lambda \cdot l_{1}\right) \cdot x\right| \geqslant \varepsilon|\lambda| \cdot|x|
$$

for $|\lambda|,|x|$ small. In certain cases, it is guaranteed that a connected family of zeroes will bifurcate from $(\lambda, x)=(0,0)$ (see [2]); we would like to find them numerically. We will reduce the question to a couple of applications of the implicit function theorem and the vector-field problem.

The standard Lyapunov-Schmidt process [26, \$2.7.4] allows us (via the implicit-function theorem) to assume that $l_{0}=0$, which we now do. In this case, $B$ must be finite dimensional, say $R^{m}$. Moreover, there are conditions on the possible values of $m$ [5], [6]. Briefly put, there exist $(n-1)$ linearly independent vector fields on $S^{m-1}$. Conversely, $m$ is a multiple of some number depending on $n$. Suppose that $m$ is an odd multiple of this number and that $n \equiv 0,1,2,4 \bmod 8$. Then bifurcation is guaranteed. Two important cases are $n=1$ and $m$ is odd, in which case we are considering Krasnosel'skii-Rabinowitz bifurcation where the relevant eigenvalues cross zero with nonzero derivative [27], [26, §3.3,3.4], and $n=2$ and $m \equiv 2 \bmod 4$, in which 
case we are considering Hopf bifurcation where the relevant eigenvalues cross the imaginary axis with nonzero derivative [6, (4)].

The case $n=m=2$ can be handled by standard implicit function theory, so we eliminate this case. For $x$ near 0 , the derivative $D_{\lambda} F(0, x): R^{n} \rightarrow R^{m}$ is approximately $l_{1} \cdot x$. In particular, if $x$ is near, but not equal to, zero, the range of $d_{\lambda} F(0, x)$ is $n$-dimensional, since our assumptions imply that $\lambda \cdot l_{1}$ is nonsingular for each $\lambda \neq 0$. Let $R_{x}^{n}$ be the range $D_{\lambda} F(0, x)$ and let $Q_{x}$ : $R^{m} \rightarrow F_{x}^{n}$ be the orthogonal projection. We consider a sphere $S_{n}$ of radius $\varepsilon>0$ in $R^{m}$. On this sphere there is a point $x_{0}$ and a point $\lambda_{0}=\lambda\left(x_{0}\right)$ where $F\left(\lambda_{0}, x_{0}\right)=0$ (since we know bifurcation occurs). By the implicit function theorem, there exists a function $x \mapsto \lambda(x)$ defined on $S_{n}$ near $x_{0}$ such that $F(\lambda(x), x)=0$. By repeated applications of the implicit function theorem and the fact that $S_{n}$ is simply connected, there exists a function $x \mapsto \lambda(x)$ defined on all of $S_{n}$ so that $F(\lambda(x), x)=0$. Let $\lambda_{1}, \ldots, \lambda_{n}$ be basis vectors in $R^{n}$ and let $l_{(i)}=\lambda_{i} \cdot l_{1}$. Then $\varphi(x)=F(\lambda(x), x)$ is independent of $l_{(i)}$ for $i=1, \ldots, n$. Consider the functions $l_{(1)}^{-1} \varphi(x), l_{(1)}^{-1} l_{2} \cdot x, \ldots, l_{(1)}^{-1} l_{n} \cdot x$ for $x \in S_{n}$. These are independent of $x=l_{(1)}^{-1} l_{(1)} \cdot x$ and each other. The Gram-Schmidt orthogonalization process turns these functions into $\rho_{m}$ vector fields on $S_{n}$. They are linearly dependent only when $\varphi(x)=0$; i.e. when there is bifurcation, and hence bifurcation points can be located by the methods of this paper.

It is not clear how efficacious this approach is for locating the bifurcating zeroes, but it is an approach.

Two generic proofs. We offer differential topology proofs of the finite-dimensional Rabinowitz bifurcation result and of L. Nirenberg's formulation of the Borsuk-Ulam Theorem. For the Rabinowitz Theorem ([27] or [26, §3.3.4]), we consider a $C^{1}$ map $f: R \times R^{n} \rightarrow R^{n}$ with $f(\mu, 0)=0$ and we want to find other solutions of $f(\mu, x)=0$. Let $S$ be the closure of the set of nontrivial zeroes-those with nonzero $x$-coordinate. Let $A(\mu)=D_{x} f(\mu, 0)$, so that $f(\mu, x)=A(\mu) \cdot x+o(|x|)$ uniformly on bounded $\mu$-intervals. Suppose that $\mu_{-}<\mu_{0}<\mu_{+}$are points in $R$ such that det $A(\mu)$ has opposite signs on $\left[\mu_{-}, \mu_{0}\right)$ and $\left(\mu_{0}, \mu_{+}\right]$(and hence $\left.\operatorname{det} A\left(\mu_{0}\right)=0\right)$. Let $C$ be the connected component of $S \cup\left\{\mu_{0}\right\}$ containing $\mu_{0}$. Indentify $R$ with $R \times\{0\}$ as a subspace of $R \times R^{n}$. The Rabinowitz Theorem states that $C$ contains some point $\mu_{1} \in R$ with $\mu_{1} \notin\left[\mu_{-}, \mu_{+}\right]$or $C$ is unbounded.

To prove it we assume it is false. We can then surround $C$ with a bounded neighborhood $\Omega$ such that $\partial \Omega \cap S$ is empty and $(\mu, 0) \in \Omega$ if and only if $\mu \in\left[\mu_{-}, \mu_{+}\right]$. We can assume that $\bar{\Omega}$ contains the set $\left[\mu_{-}, \mu_{+}\right] \times D$ for some small disk $D$ in $R^{n}$, and that near $\mu_{ \pm}$, the neighborhood $\bar{\Omega}$ contains no points not in $\left[\mu_{-}, \mu_{+}\right] \times D$. Since $\bar{\Omega}$ is compact, the values of $f$ on $\partial \Omega-\left(\left\{\mu_{ \pm}\right\} \times\right.$ $D)$ are bounded away from 0 .

Note that we can assume $f$ is smooth, because if $f$ does not satisfy the 
conclusion, we can approximate it by a smooth $f$ that also satisfies the hypotheses but not the conclusion. Since the regular points of $f$ are residual, we can choose a regular value $x$ of $f$ so that $\partial \Omega \cap f^{-1}(x)$ consists precisely of one point $p_{ \pm}$in each of $\left\{\mu_{ \pm}\right\} \times D$. Now $f^{-1}(x) \cap \bar{\Omega}$ is a one-dimensional manifold, so $p_{ \pm}$must be the two ends of an arc in $f^{-1}(x) \cap \bar{\Omega}$. On the other hand, since the domain and range of $f$ can be oriented, $f^{-1}(x) \cap \bar{\Omega}$ inherits an orientation. This orientation can be visualized as a field of unit vectors along $f^{-1}(x) \cap \bar{\Omega}$. Since $\operatorname{det} A\left(\mu_{-}\right)$and det $A\left(\mu_{+}\right)$have opposite signs, the orientations are opposite at $p_{ \pm}$; for example, the unit vectors can both be chosen to point in towards $\mu_{0}$. Thus $p_{ \pm}$cannot be the two ends of an arc, and we have a contradiction. This proves the theorem.

Consider now Nirenberg's formulation of the Borsuk-Ulam Theorem [26, \$1.1.7]. He considers a bounded open subset $X$ of $R^{n}$ symmetric about the origin and containing the origin. Let $\psi: X \rightarrow R^{n}$ be any continuous mapping such that $\psi$ is nonzero and odd (i.e. $\psi(-x)=-\psi(x)$ ) on the boundary of $X$. The theorem states that the degree of $\psi$ with respect to the origin is odd.

Nirenberg asks the following question: can an odd continuous map $\phi$ : $X \rightarrow R^{n}$ with $\phi(x) \neq 0$ for $x \in \partial X$ be approximated by an odd $C^{1}$ map such that 0 is a regular value? He does not answer the question, but points out an affirmative answer would give an easy proof of the theorem. To wit: if the answer is affirmative, let $\phi(x)=\frac{1}{2}(\psi(x)-\psi(-x))$ for $x \in X$. Approximate the odd function $\phi$ by $\phi_{1}$ as in the question. The inverse image $\phi^{-1}(0)$ must be symmetric and so consist of 0 and an even number of other points. Therefore the degree of $\phi_{1}$, which equals the degree of $\psi$, is odd.

It remains for us to answer the question. Given $\phi$, approximate it by an differentiable map $\phi_{1}$, and let $\tilde{\phi}(x)=\frac{1}{2}(\phi(x)-\phi(-x))$. Then $\tilde{\phi}$ is odd. We claim that for a dense set of matrices $A: R^{n} \rightarrow R^{n}$ the function $\phi_{A}(x)=A x$ $+\phi(x)$ has zero as a regular value. Choosing such an $A$ close enough to the zero matrix provides the desired approximation. First consider $D_{x} \phi_{A}(0)=A$ $+D_{x} \phi(0)$. The set of nonsingular matrices is generic in the set of all matrices; therefore $V=\left\{A \mid D_{x} \phi_{A}(0)\right.$ is nonsingular $\}$ is generic. Consider $F: V \times X \rightarrow$ $R^{n}$ given by $F(A, x)=\phi_{A}(x)$. The tangent space to $V \times X$ at a point $(A, x)$ is a copy of $\mathfrak{m} \times R^{n}$, where $\mathfrak{m}$ is the space of all $(n \times n)$ matrices. Let $(M, y)$ be in the tangent space at $(A, x)$. The differential DF maps $(M, y)$ to $M x+A y$ in $R^{n}$, the tangent space to $R^{n}$ at $F(A, x)$. If $x \neq 0$, for any $z \in R^{n}$, there exists an $M$ such that $M x=z$. Therefore, $F$ is a submersion (i.e. $D F$ is surjective) whenever $x \neq 0$, and hence is transverse to any submanifold. We already know that $F$ is transverse to 0 at $x=0$, therefore $F$ is transverse to 0 on all of $V \times X$. By the fundamental lemma about transversality, $\phi_{A}(x)$ is transverse to 0 for a residual set of $A$. This proves the result. 


\section{REFERENCES}

1. J. F. Adams, Vector fields on spheres, Ann. of Math. (2) 75 (1962), 603-632.

2. J. C. Alexander, Bifurcation of zeroes of parameterized functions, J. Functional Analysis (to appear).

3. Soc. (to appear).

4. J. C. Alexander and J. A. Yorke, Global bifurcation of periodic orbits, Amer. J. Math. (to appear).

5. ___ Parameterized functions, bifurcation, and vector fields on spheres (to appear).

6. Calculating bifurcation invariants as homotopy elements of the general linear group, J. Pure Appl. Algebra (to appear).

7. S. N. Chow, J. Mallet-Paret and J. A. Yorke, Finding zeroes of maps: homotopy methods that are constructive with probability one (to appear). $1-22$.

8. B. C. Eaves, Homotopies for the continuation of fixed points, Math. Programming 3 (1972),

9. $\ldots$ A short course in solving equations with PL homotopics, Non-linear Programming.

SIAM-AMS Proceedings, vol. 9, Amer. Math. Soc., Providence, R. I., 1976.

10. B. C. Eaves and H. Scarf, The solution of systems of piecewise linear equations, Math. Operations Research 1 (1976), 1-27.

11. S. Friedland, On inverse multiplicative eigenvalue problems for matrices, Linear Algebra and Appl. 12 (1975), 127-137.

12. V. Guillemin and A. Pollack, Differential topology, Prentice-Hall, Engelwood Cliffs, N. J., 1974.

13. M. Hirsch, $A$ proof of the non-retractibility of a cell onto its boundary, Proc. Amer. Math. Soc. 14 (1963), 364-365.

14. __ Differential topology, Springer-Verlag, New York, 1976.

15. D. Husemoller, Fiber bundles, Springer-Verlag, New York, 1974.

16. R. B. Kellogg, T. Y. Li and J. A. Yorke, A method of continuation for calculating a Brouwer fixed point, Fixed Points, Algorithms, and Applications (S. Karemdian, ed.), Academic Press, New York, 1977.

17. __ A constructive proof of the Brouwer fixed point theorem and computational results, SIAM J. Numer. Anal. 13 (1976), 473-483.

18. H. B. Keller, Numerical solution of bifurcation and non-linear eigenvalue problems, Applications of Bifurcation Theory, Academic Press, New York (to appear).

19. H. W. Kuhn, Simplicial approximation of fixed points, Proc. Nat. Acad. Sci. U. S. A. 61 (1968), 1238-1242.

20. T. Y. Li, A rigorous algorithm for fixed point computation (to appear).

21. T. Y. Li and J. A. Yorke, A rigorous algorithm for solving equations when existence is proved using topological degree (in preparation).

22. Y.-C. Lu, Singularity theory and an introduction to catastrophe theory, Springer-Verlag, New York, 1976.

23. G. Meyer, On solving nonlinear equations with a one-parameter operator embedding, SIAM J. Numer. Anal. 5 (1968), 739-752.

24. J. W. Milnor, Topology from the differential viewpoint, Univ. of Virginia Press, Charlottesville, Va., 1965.

25. , Morse theory, Ann. of Math. Studies, no. 51, Princeton Univ. Press, Princeton, N. J., 1963.

26. L. Nirenberg, Topics in nonlinear functional analysis, New York Univ. Lecture Notes, 1974.

27. P. Rabinowitz, Some global results for non-linear eigenvalue problems, J. Functional Analysis 7 (1971), 487-573.

28. W. C. Rheinboldt, Numerical continuation methods for finite element applications, Formulation and Computational Algorithms in Finite Element Analysis, M.I.T. Press, Cambridge, 1977. 
29. R. Saigal, Fixed point computing methods, Encyclopedia of Computer Science and Technology, Dekker, New York (to appear).

30. $\longrightarrow$ On the convergence rate of algorithms for solving equations that are based on methods of complementary pivoting, Math. Operations Research (to appear).

31. H. Scarf, The approximation of fixed points of a continuous mapping, SIAM J. Appl. Math. 15 (1967), 1328-1343.

32. S. Smale, Convergent process of price adjustment and global Newton methods, J. Math. Econom. 3 (1976), 1-14.

Department of Mathematics, University of Maryland, College Park, Maryland 20742

Institute for Physical Science and Technology, University of Maryland, College PARK, MARYLAND 20742 